\title{
Light confinement by two-dimensional arrays of dielectric spheres
}

\author{
M. López-García, J. F. Galisteo-López, ${ }^{*}$ and C. López \\ Instituto de Ciencia de Materiales de Madrid (CSIC) C/ Sor Juana Inés de la Cruz 3, 28049 Madrid, Spain
}

\author{
A. García-Martín \\ IMM-Instituto de Microelectrónica de Madrid (CNM-CSIC) c/ Isaac Newton 8, PTM, Tres Cantos, 28760 Madrid, Spain
}

(Received 20 March 2012; revised manuscript received 16 May 2012; published 25 June 2012)

\begin{abstract}
We present a study on the ability of two-dimensional close-packed arrays of submicron dielectric spheres to confine electromagnetic radiation. Substrates having different nature, either dielectric or metallic, are considered, and the evolution of the strong spatial redistribution of the total field intensity is monitored by means of numerical simulations. The physical origin of the different modes of the system is further studied by measuring the optical response of the hybrid structures and reproducing it with an analytic effective medium model. The study evidences that the use of metallic substrates provides the best scenario for enhancing light-matter interaction in this kind of system, pointing out that the hybrid modes arising from the combination of periodic dielectric lattices and metallic substrates cannot be treated as purely photonic or plasmonic.
\end{abstract}

DOI: 10.1103/PhysRevB.85.235145

PACS number(s): 42.70.Qs, 73.20.Mf, 78.66.Vs

\section{INTRODUCTION}

Two-dimensional (2D) photonic crystal (PC) slabs are nowadays acknowledged as a powerful approach to efficiently confine electromagnetic radiation in three dimensions (3D) for the visible and near-infrared part of the electromagnetic spectrum. ${ }^{1}$ Such ability has led several groups to explore the possibility to employ these systems as a means to obtain single photon sources, ${ }^{2}$ efficient lasers, ${ }^{3}$ or all-optical switches, ${ }^{4}$ to name but a few of the applications envisaged for them. But the high-optical quality of these PC comes at the cost of expensive and time-consuming fabrication techniques such as i.e., electron beam lithography or focused ion beam. In this respect self-assembly methods, which have been present in the field of PC since its early days, represent a cost-effective alternative. ${ }^{5}$ Although self-assembly has been mostly oriented toward the fabrication of 3D structures, the optical properties of monolayers of hexagonally packed dielectric spheres have been studied for decades ${ }^{6}$ and interpreted in terms of photonic band structures. ${ }^{7,8}$

Due to the low refractive index of the materials from which monodisperse submicron dielectric spheres, able to operate in the visible and infrared, are made from $(\sim 1.5)$, the light confinement properties of self-assembled 2D PC are generally not as good as their slab counterparts. Furthermore, since they require a substrate on which to rest, the compound system loses the light-confining ability. Recently, hybrid photonicplasmonic systems have been proposed as a means to improve light confinement in such self-assembled structures. In these systems monolayers of dielectric spheres are deposited on metal substrates, usually gold or silver, giving rise to the existence of hybrid modes where the electromagnetic field is located within the spheres and/or close to the metal surface, depending on the photonic or plasmonic character of the mode respectively. ${ }^{9-11}$ The large electromagnetic field redistribution taking place in this kind of systems can be used to strongly modify the spontaneous emission of internal light sources, ${ }^{11}$ improve the efficiency of thin-film solar cells, ${ }^{12}$ or as chemical sensors. ${ }^{13}$ Further, the dependence of their optical response with the structural parameters of the dielectric monolayer and the optical constants of the metal substrate can be used to precisely tune the spectral position and quality factor of the different modes of the system. ${ }^{14,15}$

In this article we present a thorough study of the light confinement efficiency of 2D PC slabs made from dielectric spheres. The optical response as well as the total field intensity spatial distribution of the different modes present in monolayers of dielectric spheres is studied by means of finite-difference time-domain (FDTD) simulations. Special attention is devoted to the effect on the optical response of the nature of the substrate on which the monolayer rests on. We discuss the evolution of the different modes present in an ideal free-standing monolayer as a dielectric substrate with varying refractive index is introduced. Further, we consider the effect of introducing a surface plasmon polariton (SPP) supporting metallic substrate. Finally, we experimentally study the dispersion relation of the latter samples and reproduce it with an empty lattice model, which provides us valuable information regarding the character of the different modes present in the system.

\section{FREE-STANDING MONOLAYER}

We first consider the simplest scenario, corresponding to a free-standing monolayer of dielectric spheres in vacuum. On a first approximation this system can be considered as a $2 \mathrm{D}$ PC slab in which propagation in the plane will be governed by Bragg diffraction, while confinement in the vertical direction will be provided by total internal reflection. Hence, one expects that, in analogy with the slab case, two types of modes will be available: those lying above (leaky) and below (guided) the light line of the system. Only the first ones will be available in far-field reflection/transmission experiments like those considered in this work, and therefore we will focus our attention on them.

In order to simulate the optical response of this kind of structures, we have performed FDTD simulations. ${ }^{16}$ This has allowed us to obtain both, reflectance spectra as well as total field intensity spatial distributions. Our computational 
(a)

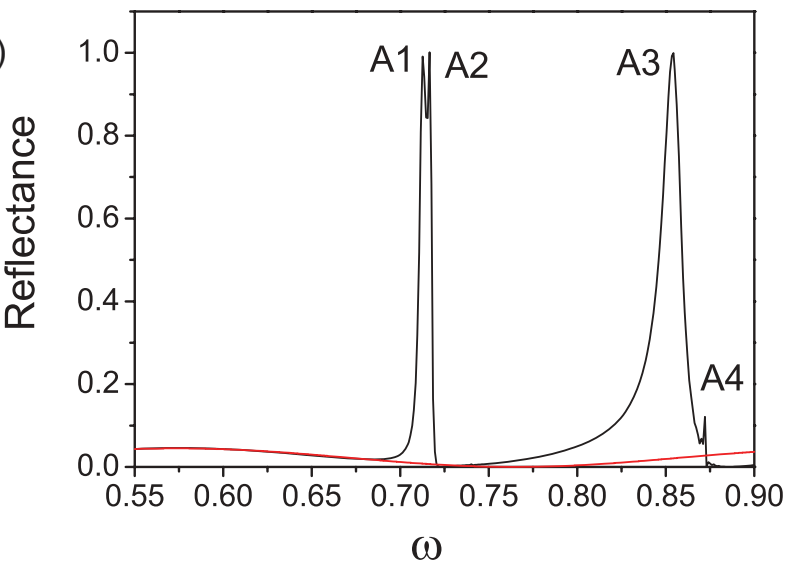

(b)

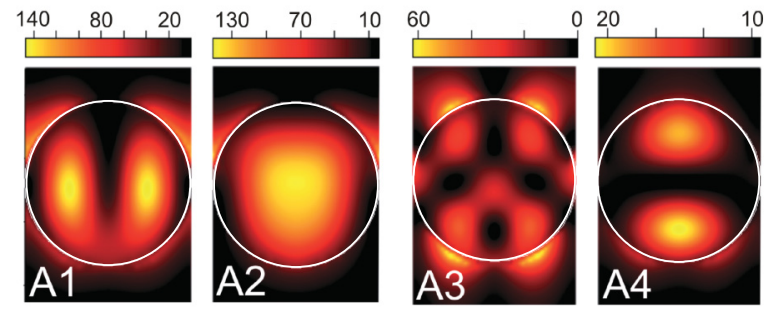

FIG. 1. (Color online) (a) Calculated reflectance spectrum for a monolayer of 520-nm polystyrene spheres surrounded by air. Red (medium gray) line shows reflectance for a 475-nm slab with refractive index 1.24. (b) Total field intensity spatial distribution of the different electromagnetic modes of the system.

cell has the triangular unite cell dimensions in the $x y$ plane of periodicity and a varying dimension in the $z$ direction depending on whether the monolayer is free-standing or resting on a substrate. A sufficiently fine grid $(\geqslant 40$ points per wavelength) and long simulation time $(\geqslant 1 \mathrm{ps})$ were used in order to correctly simulate the structures resting on metal substrates.

Figure 1 shows a reflection spectrum at normal incidence for a free-standing monolayer of polystyrene spheres $(\mathrm{n}=$ 1.59) having a diameter $d_{\text {sph }}=520 \mathrm{~nm}$. Results are presented in terms of reduced frequency $\omega=\sqrt{3} \cdot \mathrm{d}_{\mathrm{sph}} /(2 \cdot \lambda)$. Two kinds of optical features can be appreciated in the spectrum. On the one hand, a spectrally broad oscillation with small $(<10 \%)$ amplitude associated with Fabry-Perot resonances due the finite size of the monolayer in the vertical direction. Such oscillations can be accounted for assuming a 475-nm-thick film with refractive index 1.25 , close to the sphere diameter and volume averaged refractive index of the monolayer. On the other hand, there are a number of spectrally sharp peaks (A1-A4) corresponding to the leaky modes of the sample in this spectral range. Such modes are commonly observed as peaks/dips in reflectance/transmittance experiments. ${ }^{17}$ In order to gain insight into the nature of the modes, we have also obtained the total field intensity associated with the different frequencies at which they take place. Figure 1(b) shows the field intensity associated with leaky modes A1-A4, evidencing a strong concentration of the electromagnetic field within or in the surroundings of the spheres. The exact shape of the profile depends on the order of the mode considered, and it is strongly related to Mie resonances of isolated spheres as well as to the coupled array. ${ }^{18}$

\section{DIELECTRIC SUBSTRATE}

The above results evidence that a free-standing monolayer is able to effectively confine electromagnetic radiation through the different modes arising as a consequence of multiple scattering by individual spheres. But, from a practical point of view, it is not straightforward to fabricate or manipulate such free-standing structures working in the visible-infrared spectral range, and hence a substrate, normally a dielectric, must be used. As a matter of fact, free-standing monolayers of submicron dielectric spheres have not been reported in the literature to the best of our knowledge.

Following the PC slab analogy, introducing a dielectric substrate with a refractive index $>1$ will inevitably reduce the confinement capability of our 2D structure. ${ }^{8,19}$ Here, we want to consider in a systematic manner the effect of substrate refractive index on the light confinement properties of the dielectric monolayer. We start by calculating the optical response of monolayers of polystyrene spheres resting on dielectric substrates of increasing refractive index. Figures 2(a)-2(c) show normal incidence reflectance spectra for samples with substrates having a different refractive index. Here, we can see how an increase of the refractive index of
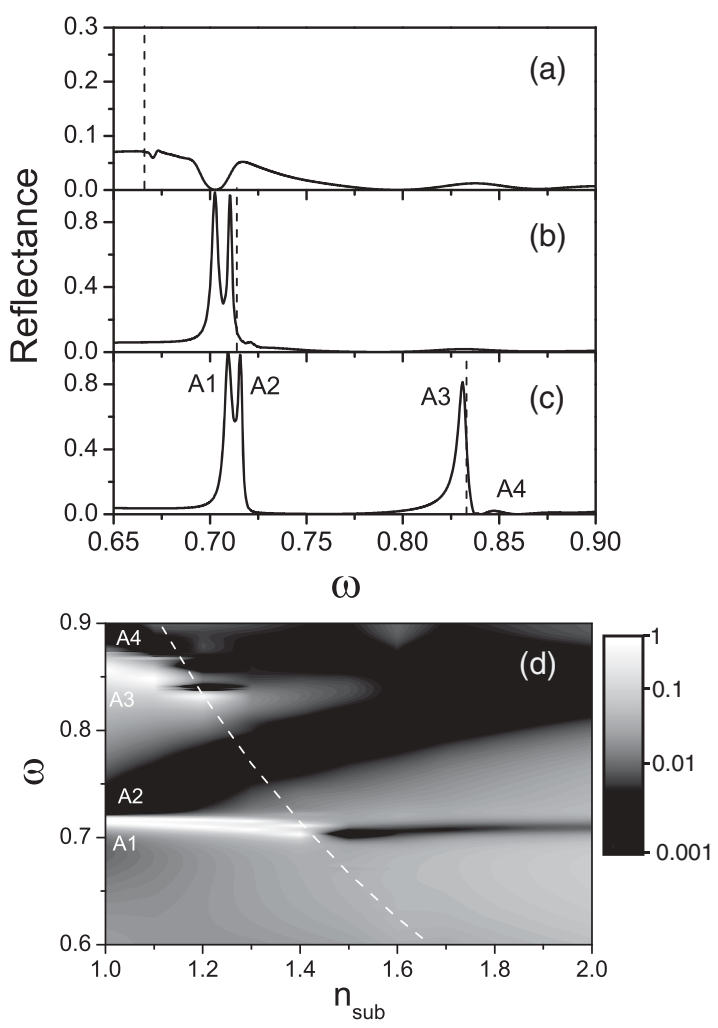

FIG. 2. Calculated reflectance spectra of monolayers of polystyrene spheres deposited on dielectric substrates of different refractive index: 1.5 (a), 1.4 (b), and 1.2 (c). (d) Contour plot showing reflectance as a function of substrate refractive index in a logarithmic scale. Dashed line shows the onset of out-of-plane diffraction. 
the substrate introduces a spectral shift of the peaks. These are also broadened, and their amplitude decreased until they eventually vanish, evidencing the introduction of radiative losses via the substrate. The latter effect takes place first for the high-energy modes. In order to understand this, we can establish an analogy with a conventional planar dielectric waveguide $^{20}$ for which the higher the order of a given mode, the higher its energetic position and the lower its modal effective refractive index. Hence, reflectance peaks vanish as the effective index of the mode approaches that of the substrate, evidencing the introduction of radiative losses. This effect takes place for $\mathrm{n}_{\text {sub }} \approx 1.4$ (where $\mathrm{n}_{\text {sub }}$ is the refractive index of the substrate), close to the effective index of the monolayer, taken as the volume average of the indices of the spheres and the surrounding air. Some authors ${ }^{21}$ have reported that this approach is appropriate for PC slabs, and we demonstrate here that for a monolayer of spheres the effect of increasing $\mathrm{n}_{\text {sub }}$ can be qualitatively explained in these terms. Following this analogy, the spectral shift of the modes is also present in guided optics theory and known as the isolating oxide substrate effect. $^{20}$

Together with the leakage to the substrate as $\mathrm{n}_{\text {sub }}$ increases, one must also take into account the presence of out-ofplane diffraction, which diminishes light confinement. For a free-standing monolayer, the onset of diffraction takes place for $\omega_{\text {diff }}=1$. But as we increase the substrate refractive index, in the convention we use for $\omega$, this onset changes to $\omega_{\text {diff }}=1 / n_{\text {sub }}$. In Figs. 2(a)-2(c) we have indicated the onset of diffraction for the different substrates as vertical dashed lines. Here it is evident that out-of-plane diffraction is the most likely cause for the loss of confinement. For substrate indices above 1.5 , the onset of diffraction takes place for frequencies $\omega_{\text {diff }}<$ 0.67 and hence out of the spectral range under consideration. For such indices, the optical response is essentially that of a dielectric film, with Fabry-Pérot oscillations of increasing amplitude as the refractive index contrast between the substrate and the spheres increases. Overimposed on these oscillations are small dips reminiscent of the modes of the system. To better appreciate the latter, Fig. 2(d) shows a contour plot with reflectance for increasing substrate refractive indices $\mathrm{n}_{\text {sub }}=$ 1-2. Here we can see how below the onset of out-of-plane diffraction (shown as a dashed line), the different modes of the system appear as white lines corresponding to the reflectance peaks shown in Figs. 2(a)-2(c). Above the diffraction limit, the different modes appear as black lines associated with dips over imposed on Fabry-Pérot oscillations, evidencing the loss of confinement.

To further evidence the loss of confinement, we will next consider the spatial distribution of the total field intensity for $\mathrm{n}_{\text {sub }}=1.2$ and 1.4. Only modes A1-A3 are considered since A4 loses all confinement for $\mathrm{n}_{\text {sub }}>1.15$. Here, it becomes evident that for $\mathrm{n}_{\text {sub }}=1.2$ the onset of diffraction takes place close to the spectral position of A3, and hence no field confinement takes place for this frequency. Analogously, for $\mathrm{n}_{\text {sub }}=1.4$ the onset diffraction closely matches $\mathrm{A} 2$, which translates into a drop in the total field intensity associated with this mode. Thus, the combination of reflectance spectra with spatial field distributions show how the use of dielectric substrates is highly detrimental for light confinement in monolayers of dielectric spheres. (a)
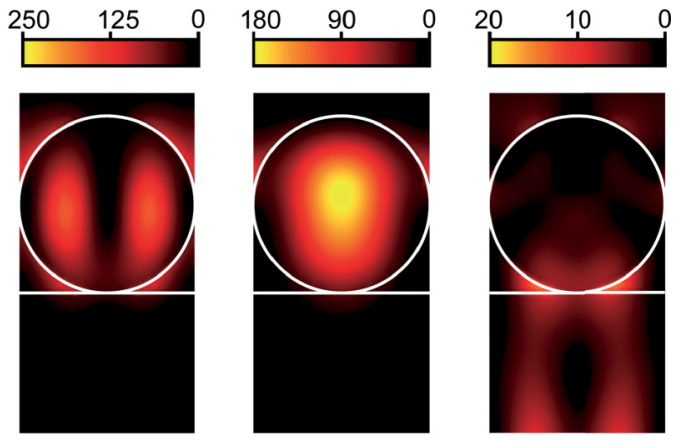

(b)

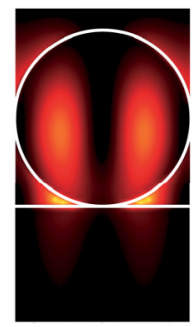

A1

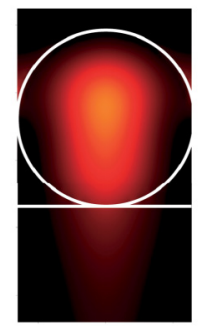

A2

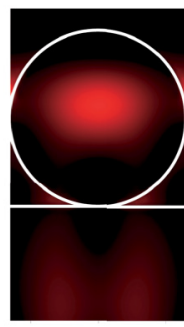

A3
FIG. 3. (Color online) Total field intensity spatial distribution associated with the A1, A2, and A3 modes of a monolayer of dielectric spheres deposited on dielectric substrates having a refractive index of 1.2 (a) and 1.4 (b).

\section{METALLIC SUBSTRATE}

As pointed out in the introduction, several works have recently shown how combining monolayers of dielectric spheres with metallic substrates can strongly confine electromagnetic radiation via the introduction of hybrid modes. ${ }^{9-15}$ This becomes evident when we consider the optical response of a monolayer of polystyrene spheres deposited on a gold substrate (as will be seen in Fig. 4). Here the different modes of the system appear as dips over a nearly unit reflectance background, similar to that of a gold film. The fact that we obtain dips rather than peaks, as in the case of a dielectric substrate, can be explained as for $2 \mathrm{D}$ slab $\mathrm{PC},{ }^{17}$ where the spectral shape of the modes is the result of the combination of a resonant state with a continuum of modes, which gives rise to the characteristic Fano line shape observed. The enhanced confinement of the electromagnetic field can be noted if one compares the values on the scale bars of the contour plots with those of Figs. 2 and 3. Such enhancement is up to an order of magnitude larger compared to the ideal free-standing case and hence cannot just be explained by a reduction of radiative losses by the presence of a reflecting substrate. For the case of the G2 and G4 modes, the spatial distribution of the field intensity is maintained and the origin of the enhancement is related to constructive interference, similar to the case of the so-called 2.5 PC, ${ }^{22}$ where light confinement is improved by the introduction of a dielectric reflector below a slab 2D PC. For the G1 and G3 modes, we can see how the total field intensity changes if compared to the free-standing system, and it is mostly concentrated close to the metal substrate, giving them its "plasmonic" character. The close vicinity of the metallic substrate is probably responsible for the low quality factor $\mathrm{Q}$ (given by $\left.\omega_{\mathrm{c}} / \Delta \omega\right)$ associated with these modes $\left(\mathrm{Q}_{\mathrm{G} 1}=125\right.$, 


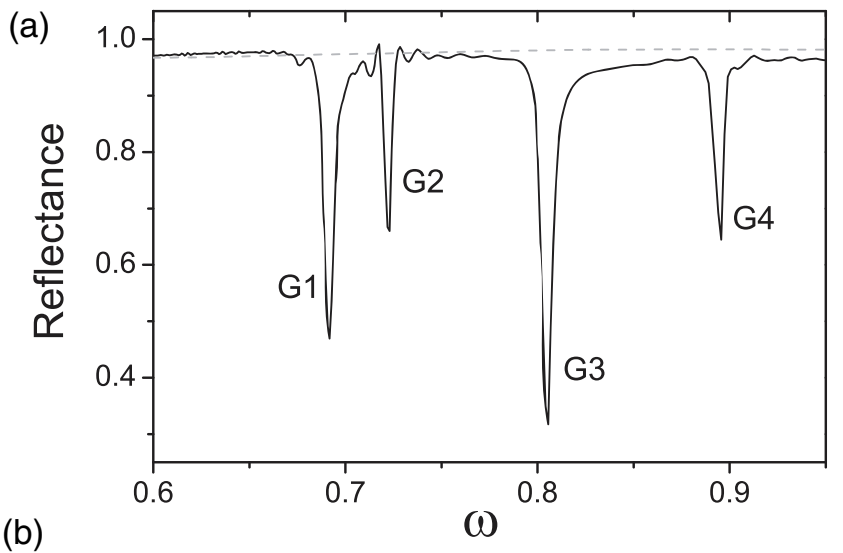

(b)

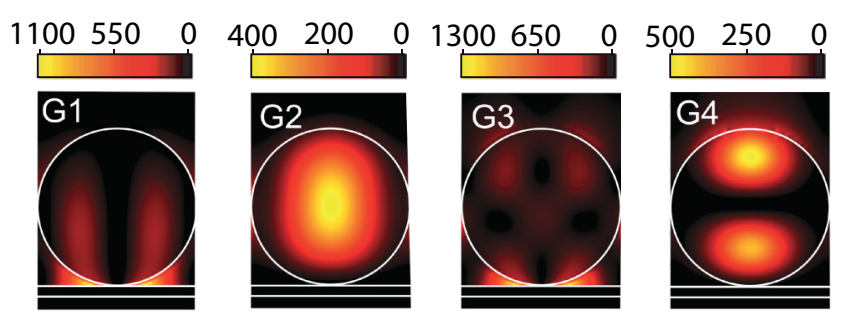

FIG. 4. (Color online) (a) Reflectance spectra for a monolayer of dielectric spheres of $1-\mu \mathrm{m}$ diameter deposited on a 60 -nm gold film (solid black line) together with the reflectance of the bare film (dashed gray line). (b) Total field intensity for the different modes selected in (a).

$\left.\mathrm{Q}_{\mathrm{G} 3}=130\right)$ when compared to the photonic ones $\left(\mathrm{Q}_{\mathrm{G} 2}=215\right.$, $\mathrm{Q}_{\mathrm{G} 4}=188$ ), due to the losses introduced by gold.

In order to gain insight into the physical nature of the different modes, we have fabricated samples like the simulated one and optically characterized them by means of angle and polarization resolved reflectance (see Ref. 11 for more information on fabrication procedure and optical characterization). Experimental results for monolayers of 520-nm diameter polystyrene spheres deposited on a thin (60-nm) silver film as will be shown in the left panels of Figs. 5(a) and 5(b). Here the angular evolution of the different modes of the system appear as dark streaks over a bright background, corresponding to the angular evolution of the dips observed in the normal incidence spectrum of Fig. 4 for normal incidence. In order to reproduce the dispersion of the G1-G3 modes, visible in the experimental data, we have assumed an effective medium model. In this approach we consider two different types of modes. On the one hand, those having a "photonic" character with most of the field intensity located within the sphere are modeled considering an effective dielectric with refractive index $n_{\mathrm{WG}}$ such that

$$
\omega=\frac{\sqrt{3} r}{2 \pi n_{\mathrm{WG}}} k
$$

where $r$ is the sphere radius. On the other hand, those having a "plasmonic" character with the field intensity concentrated close to the metal surface are modeled using the dispersion relation of a SPP

$$
k_{\mathrm{SPP}}=\frac{2 \pi}{\sqrt{3 r}} \omega \sqrt{\frac{\varepsilon_{1} \varepsilon_{2}}{\varepsilon_{1}+\varepsilon_{2}}},
$$

(a)
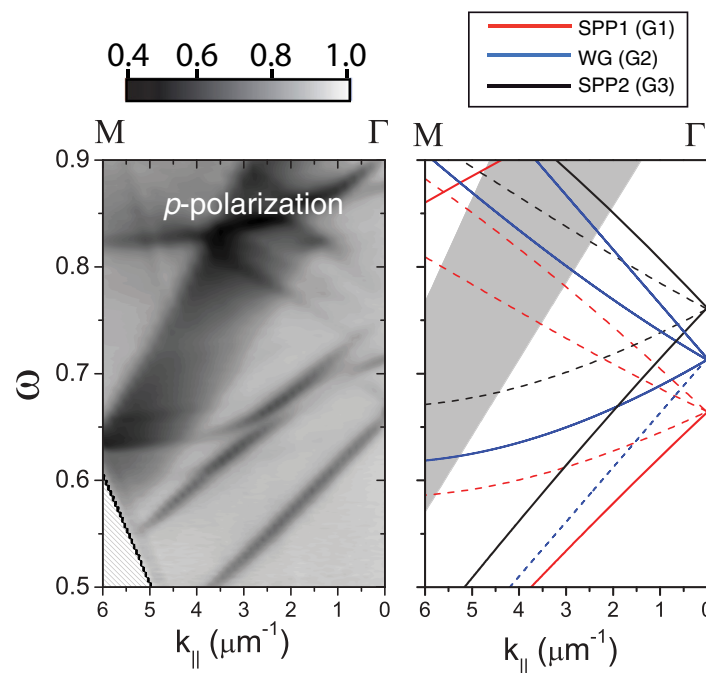

(b)
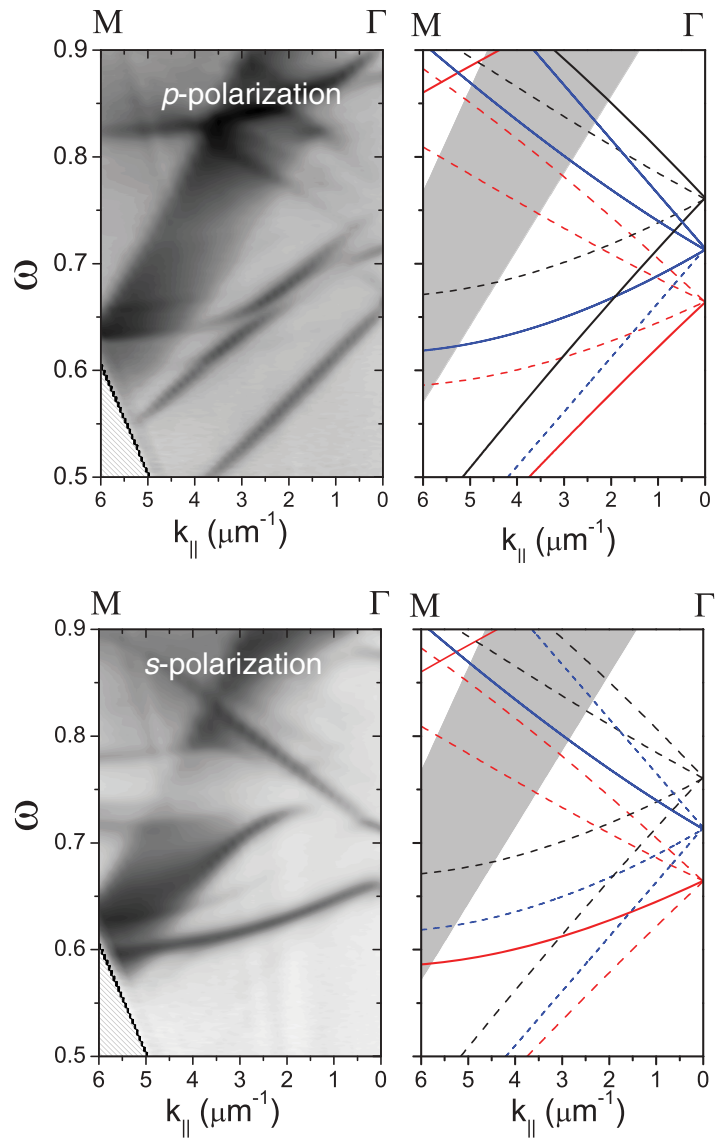

FIG. 5. (Color online) Angle-resolved reflectance for a monolayer of polystyrene spheres grown on a $60-\mathrm{nm}$ silver film measured along the $\Gamma \mathrm{M}$ direction in reciprocal space for $p$ - (a) and $s$ - (b) polarized light. Right panels show dispersion relations calculated using an effective medium model (see text). Solid lines are highlighted branches of the calculated dispersion relation, which reproduce experimental data.

where $\varepsilon_{1}$ and $\varepsilon_{2}$ are the dielectric constants of silver and an effective dielectric medium, respectively. In both cases, the dispersion relation is folded back into the first Brillouin zone, and diffraction by the different reciprocal lattice vectors in the plane of periodicity of the monolayer is considered. The obtained dispersion relations are plotted in the right panels of Fig. 5. The values of $\mathrm{n}_{\mathrm{WG}}(1.4)$, and $\varepsilon_{2}$ for the G1 (1.54) and G3 (2.04) modes were obtained by matching the spectral position of the G1-G3 modes at normal incidence with the above analytical expressions.

In the comparison of Fig. 5, we have highlighted the branches of the calculated dispersion that better reproduce the experimental data (thick solid lines). Here, it is evident that, even though the values used for $\mathrm{n}_{\mathrm{WG}}$ and $\varepsilon_{2}$ were obtained by fitting the experimental spectra at normal incidence, the agreement is also maintained for large angles of incidence. Besides such good agreement, it must be noted that the distinction usually made between "plasmonic" and "photonic" modes in this kind of systems is a simplification of the problem evidenced, for instance, by the fact that modes having a 
plasmonic character can be excited with $s$-polarized light, something not allowed in purely plasmonic resonances. ${ }^{23}$ Further theoretical work, such as the evolution of the field intensity for off-normal angles of incidence, will probably shed some more light on the physical origin of the different modes of these systems.

\section{CONCLUSION}

We have studied how close-packed 2D arrays of submicron dielectric spheres can efficiently confine electromagnetic radiation. We have analyzed the evolution of such confinement from the ideal case of the free-standing structure to the more realistic scenarios of arrays resting on dielectric and metallic substrates, finding how the latter is the most favorable case. In order to do so, we have employed FDTD simulations that have allowed us to track both, the reflectance as well as the total field intensity, showing how the different hybrid modes present in the metallodielectric structure have their origin in the free-standing one. Finally, we have experimentally studied the dispersion relation of the hybrid system and successfully reproduced it with an effective medium analytical model, which also evidences that further theoretical work will be needed to gain deeper understanding into the physical origin of these modes.

\section{ACKNOWLEDGMENTS}

M. López-García was supported by the FPI Ph.D. program from the MICINN. This work was supported by the Spanish MICINN CSD2007-0046 (Nanolight.es) and MAT200907841 projects; Comunidad de Madrid S2009/MAT-1756 (PHAMA) program and EU Nanophotonics4Energy (Contract No. 248855). A. García-Martín also acknowledges financial support from the Spanish MICINN ("MAGPLAS" 312 MAT2008-06765-C02-01/NAN, Funcoat Consolider Ingenio 2010 CSD2008-00023 and "MAPS" MAT2011-29194c02-01) and European Comission (NMP3-SL-2008-214107Nanomagma) projects.
*Corrresponding author: galisteo@icmm.csic.es

${ }^{1}$ M. Notomi, Rep. Prog. Phys. 73, 096501 (2010).

${ }^{2}$ A. Schwagmann, S. Kalliakos, I. Farrer, J. P. Griffiths, G. A. C. Jones, D. A. Ritchie, and A. J. Shields, Appl. Phys. Lett. 99, 261108 (2011).

${ }^{3}$ S. Matsuo, A. Shinya, T. Kakitsuka, K. Nozaki, T. Segawa, T. Sato, Y. Kawaguchi, and M. Notomi, Nat. Photon. 4, 648 (2010).

${ }^{4}$ K. Nozaki, T. Tanabe, A. Shinya, S. Matsuo, T. Sato, H. Taniyama, and M. Notomi, Nat. Photon. 4, 477 (2010).

${ }^{5}$ J. F. Galisteo-López, M. Ibisate, R. Sapienza, L. S. FroufePérez, A. Blanco, and C. López, Adv. Mater. 23, 30 (2011).

${ }^{6}$ M. Inoue, K. Ohtaka, and S. Yanagawa, Phys. Rev. B 25, 689 (1982).

${ }^{7}$ H. T. Miyazaki, H. Miyazaki, K. Ohtaka, and T. Sato, J. Appl. Phys. 87, 7152 (2000)

${ }^{8}$ Y. Kurokawa, H. Miyazaki, and Y. Jimba, Phys. Rev. B 69, 155117 (2004).

${ }^{9}$ R. M. Cole, Y. Sugawara, J. J. Baumberg, S. Mahajan, M. Abdelsalam, and P. N. Bartlett, Phys. Rev. Lett. 97, 137401 (2006).

${ }^{10}$ L. Shi, X. Liu, H. Yin, and J. Zi, Phys. Lett. A 37, 1059 (2010).

${ }^{11}$ M. López-García, J. F. Galisteo-López, A. Blanco, J. SánchezMarcos, C. López, and A. García-Martín, Small 6, 1757 (2010).
${ }^{12}$ J. Grandidier, D. M. Callahan, J. N. Munday, and H. A. Atwater, Adv. Mater. 23, 1272 (2011).

${ }^{13}$ X. Yu, L. Shi, D. Han, J. Zi, and P. V. Braun, Adv. Funct. Mater. 20, 1910 (2010).

${ }^{14}$ M. López-García, J. F. Galisteo-López, A. Blanco, C. López, and A. García-Martín, Adv. Funct. Mater. 20, 4338 (2010).

${ }^{15}$ J. F. Galisteo-López, M. López-García, C. López, and A. GarcíaMartín, Appl. Phys. Lett. 99, 083302 (2011).

16"FDTD Solutions" software from Lumerical Inc. (www.lumerical.com).

${ }^{17}$ S. Fan and J. D. Joannopoulos, Phys. Rev. B 65, 235112 (2002).

${ }^{18}$ H. T. Miyazaki and K. Ohtaka, Phys. Rev. B 58, 6920 (1998).

${ }^{19}$ Y. Kurokawa, H. Miyazaki, and Y. Jimba, Phys. Rev. B 65, 201102 (2002).

${ }^{20}$ D. L. Lee, Electromagnetic Principles of Integrated Optics (Wiley, New York, 1986).

${ }^{21}$ D. Gerace and L. C. Andreani, Phys. Rev. E 69, 056603 (2004).

${ }^{22}$ P. Viktorovitch, B. Ben Bakir, S. Boutami, J.-L. Leclerq, X. Letartre, P. Rojo-Romero, C. Seassal, M. Zussy, L. Di Cioccio, and J.-M. Fedeli, Laser Photon. Rev. 4, 401 (2010).

${ }^{23}$ H. Raether, Surface Plasmons on Smooth and Rough Surfaces and on Gratings (Springer-Verlag, Berlin, 1988). 